\title{
Effects of a metallic implant on radiotherapy planning treatment- experience on a human cadaver
}

\author{
Clara Le Fèvre ${ }^{1,2}$, David Brinkert ${ }^{3}$, Inès Menoux ${ }^{1}$, Florent Kuntz ${ }^{4}$, Delphine Antoni ${ }^{1,2}$, Ziad El Bitar ${ }^{2}$, \\ Alicia Thiery ${ }^{5}$, Robin Fabbro ${ }^{6}$, Georges Noël ${ }^{1,2}$ \\ ${ }^{1}$ Department of Radiotherapy, ICANS, Institut Cancérologie Strasbourg Europe, 67200 Strasbourg Cedex, France; ${ }^{2}$ Strasbourg University, CNRS, \\ IPHC UMR 7178, Centre Paul Strauss, UNICANCER, 67000 Strasbourg, France; ${ }^{3}$ Department of Orthopedic Surgery and Traumatology, \\ Hautepierre University Hospital, 67098 Strasbourg Cedex, France; ${ }^{4}$ Aerial Technology Resource Center, 67400 Illkirch Graffenstaden, France; \\ ${ }^{5}$ Department of Public Health, ICANS, Institut Cancérologie Strasbourg Europe, 67200 Strasbourg Cedex, France; ${ }^{6}$ Department of Medical Physics, \\ ICANS, Institut Cancérologie Strasbourg Europe, 67200 Strasbourg Cedex, France \\ Contributions: (I) Conception and design: C Le Fèvre, G Noël; (II) Administrative support: C Le Fèvre, G Noël, D Antoni; (III) Provision of study \\ materials or patients: C Le Fèvre, G Noël, D Brinkert, F Kuntz; (IV) Collection and assembly of data: C Le Fèvre, G Noël, I Menoux, A Thiery; (V) \\ Data analysis and interpretation: All authors; (VI) Manuscript writing: All authors; (VII) Final approval of manuscript: All authors. \\ Correspondence to: Clara Le Fèvre, MD, MSc. Department of radiotherapy, ICANS, Institut Cancérologie Strasbourg Europe, 17 rue Albert Calmette, \\ 67200 Strasbourg Cedex, France. Email: clefevre@strasbourg.unicancer.fr.
}

Background: Metallic implants (MIs) complicate radiotherapy planning. Several studies have worked on tissue-equivalent phantoms as experimental models to estimate dose distributions in this context. The application of these results to clinical practice remains disputable because the inhomogeneity of human tissue densities is a difficult factor to integrate into dose calculation software. In this work, we evaluate the impact of human tissue inhomogeneities by assessing the discrepancies between treatment planning system (TPS) dose calculations and measured delivered doses on a human cadaver with hip prostheses.

Methods: A total of 143 alanine dosimeters were positioned in contact with the prostheses (bones group), soft tissues (soft tissues group), skin surfaces (skin group) and natural cavities (cavities group) of a human cadaver. The planning target volume (PTV) corresponded to a standard endometrial cancer treatment. The irradiation was performed with $6 \mathrm{MV}$ X-ray tomotherapy at the one fraction-dose of $10 \mathrm{~Gy}$.

Results: A total of 140 dosimeters were analyzed. After applying a temperature correction coefficient to the measured doses, the global analysis of all dosimeters showed a significant difference between the calculated doses and the measured doses $(\mathrm{P}<0.001)$. For dosimeters of the bones, soft tissues, skin and cavities groups, this difference was also significant $(\mathrm{P}<0.001$ for each group). The mean measured doses were $21.9 \%$ lower than the mean calculated doses in the global analysis and $17.0 \%, 21.2 \%, 33.0 \%$ and $19.0 \%$ lower for the bones, soft tissues, skin and cavities groups, respectively.

Conclusions: This study showed that the received doses were significantly lower than the calculated doses and suggested the need to improve the understanding of this discrepancy.

Keywords: Alanine dosimeter; dosimetry; human cadaver; metallic implant (MI); radiation therapy

Submitted Sep 03, 2019. Accepted for publication Jan 14, 2020.

doi: $10.21037 /$ cco.2020.01.09

View this article at: http://dx.doi.org/10.21037/cco.2020.01.09

\section{Introduction}

Approximately $4 \%$ of patients treated with radiation therapy for cancer have metallic implants (MIs), and among them, two percent have hip prostheses (1). Hip prostheses are composed of high $\mathrm{Z}$ materials (2). These materials create an inhomogeneity in the vicinity of the low $\mathrm{Z}$ tissues composing the human body because of the density difference, atomic number disparity and variation 
of attenuation index (3). This inhomogeneity obviously unfavorably impacts all the steps of a radiation treatment.

To calculate dose deposits, treatment planning systems (TPS) use the electron density of the tissues from the kilovoltage computed tomography $(\mathrm{kV}-\mathrm{CT})$ based on a Hounsfield unit (HU)-to-electron density conversion curve $(4,5)$. This conversion curve could be inaccurate if the HU window is not extended to the highest HUs, as is required to characterize MIs. Moreover, MIs modify normal tissue images by introducing many artifacts, which affect the expected electron densities of the tissues located downstream of the beam entry $(4,6)$. These modifications hinder the definition of the exact range of target volumes and organs at risk (OARs) and compromise the delineation and dose calculations surrounding and at a distance from the MIs (7). Miscalculations lead to inaccuracies in the delivered dose and could alter the efficacy of the treatment (1).

There is no consensus on the management of these MI interactions. To approach the exact dose distribution in the presence of MIs, many studies used tissues-equivalent phantoms or water tanks. However, extrapolation of their results to clinical practice was challenging because they did not integrate the density inhomogeneity of body tissues into their models. Therefore, using a more realistic model closer to clinical practice appeared relevant to analyzing the dose distribution in the presence of an MI $(8,9)$. At this time, no study has been conducted on human cadavers for the radiotherapy of pelvic cancer in the presence of MIs even though hip prostheses could impact prostate, rectum, bladder or gynecological cancer irradiation.

The purpose of this study was to determine the reliability of the TPS dose calculations for pelvic irradiation with TomoTherapy ${ }^{\circledR}$ (Accuray Inc. ${ }^{\circledR}$, Sunnyvale, CA, USA) in the presence of MIs using a human cadaver with bilateral hip prostheses. To achieve this objective, we compared calculated doses with measured doses obtained from alanine dosimeters inserted in the cadaver.

\section{Methods}

This study received institutional ethics board approval from the research committee of the comprehensive cancer center.

\section{Human cadaver}

The Morphology and Normal Anatomy Institute of the University supported the experimental manipulations and provided a frozen adult woman cadaver (body donation) with bilateral hip prostheses. The components of the prostheses were unknown.

\section{Alanine dosimeters}

Alanine dosimeters are small pellets $4.0 \pm 0.05 \mathrm{~mm}$ in diameter, with a height of $2.3 \pm 0.1 \mathrm{~mm}$, with a mass of $37.75 \pm 0.06 \mathrm{mg}$ and that contain $93 \%$ amino-acid L-alanine. A MiniScope MS 400 ESR spectrometer (Magnettech, Germany), based on electron paramagnetic resonance (EPR), was used for alanine dosimeter analyses. Alanine dosimeters were positioned sequentially inside a quartz glass tube for the analysis. Two scans were performed with a $90^{\circ}$ tube rotation to remove the possible heterogeneities or anisotropies in the alanine dosimeters (10). Measurements of the alanine dosimeters were performed on two different spectrometers and demonstrated no significant differences (data not shown).

To improve the accuracy of the measurements and induce sufficient free radicals for a measurable reliable dose, the alanine dosimeters were pre-irradiated by a ${ }^{137} \mathrm{Cs}$ $\gamma$ irradiator (Biobeam GM8000, GSM Gmbh, Leipzig, Germany) with a dose of 2 Gy at a dose rate of $3.4 \mathrm{~Gy} / \mathrm{min}$, as recommended in our laboratory protocol.

Because the temperature during irradiation influences dose measurement values and the stability of free radicals $(10-12)$, we measured the dose received by the dosimeters at three different temperatures $-22,+4$ and $+21^{\circ} \mathrm{C}$ after a $10 \mathrm{~Gy}$ irradiation was performed with a Novalis $\mathrm{TX}^{\mathrm{TM}}$ linear accelerator (Varian Medical Systems ${ }^{\circledR}$, Palo Alto, CA, USA) with $15 \mathrm{MV} \mathrm{X}$-ray beams, allowing us to design a dosetemperature linear-regression curve (data not shown) and to calculate a corrective factor of $+0.18 \% /{ }^{\circ} \mathrm{C}(0.07 ; 0.28)$.

Humidity influences the stability of free radicals and dose measurements values $(10,11)$. Therefore, once the preirradiation was completed, the dosimeters were inserted into individually numbered, polyethylene, watertight blisters to preserve them from wet cadaver tissues (Figure 1A). To facilitate surgical implantation, the dosimeters were grouped together with waterproof adhesive tape to form tabs (Figure 1B). Eighteen tabs of seven dosimeters spaced $2 \mathrm{~cm}$ apart were prepared and positioned inside cadaver tissues. For natural cavities, the dosimeters were taped on catheters (Figure 1C). For the bladder, five dosimeters were placed on a urinary catheter $1 \mathrm{~cm}$ apart. For the rectum and vagina, six dosimeters for each cavity were situated on a rectal catheter $1 \mathrm{~cm}$ apart for the first four and $2 \mathrm{~cm}$ apart for the last two. To detect the location of the tabs and catheters on 


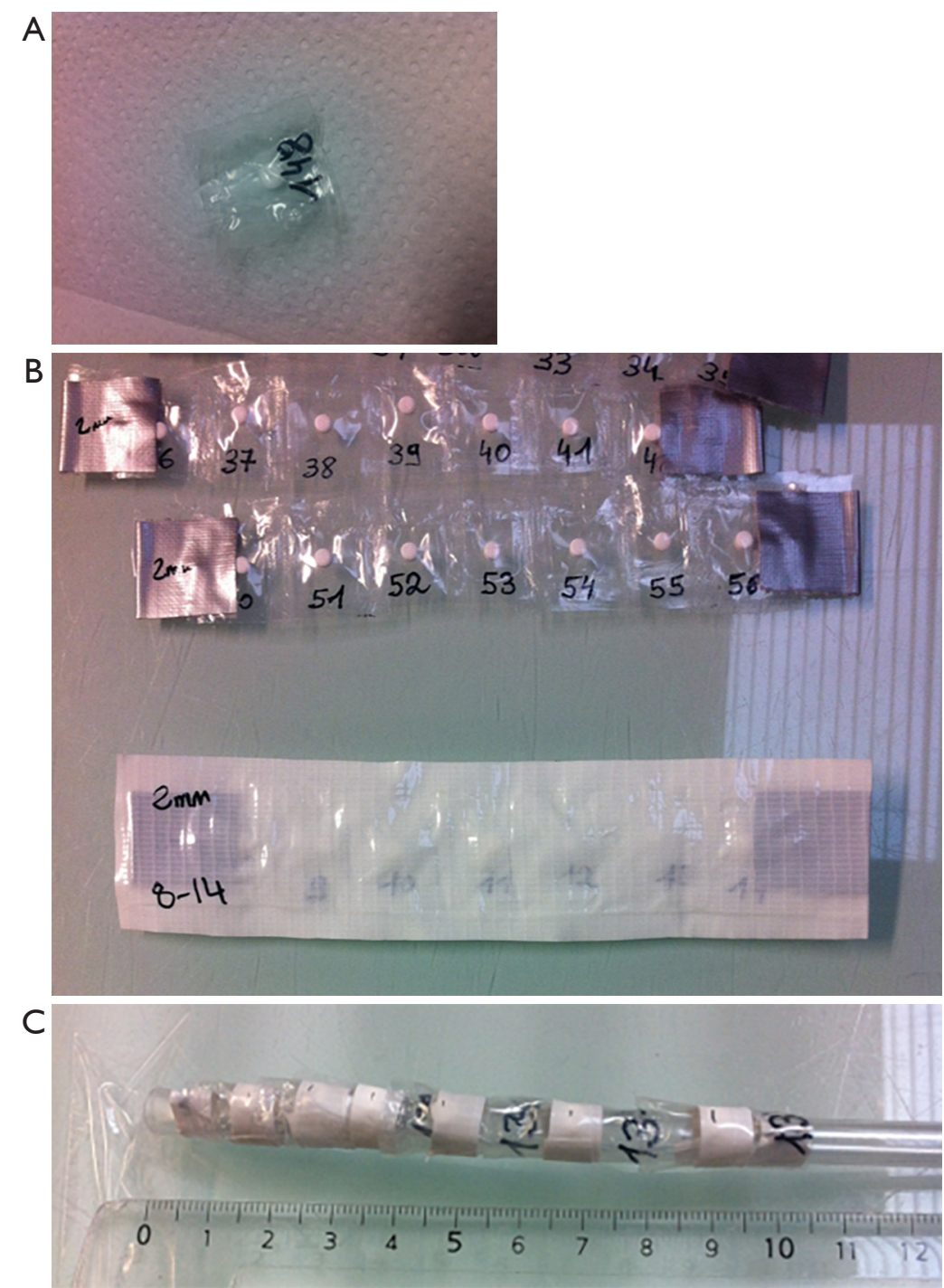

Figure 1 Alanine dosimeters' conditioning. (A) Alanine dosimeter in its individual watertight blister; (B) alanine dosimeters assembled with adhesive tape to create tabs; (C) alanine dosimeters taped on the catheter.

the dosimetric $\mathrm{kV}-\mathrm{CT}$ slices, lead markers (3 mm diameter) were placed on every tab or catheter $1 \mathrm{~cm}$ from where each dosimeter ends. To test the noninterference of adhesive tape on the absorbed dose, the dosimeters were irradiated with the $\gamma$ irradiator with 10 Gy with or without the surrounding adhesive tape. No difference in the dose was measured between the two conditions (data not shown).

The tabs and catheters, which included 143 alanine dosimeters, were positioned surgically or manually in the thawing cadaver as follows: eight tabs in contact with prostheses (bones group), six in soft tissues (soft tissues group) and four on the skin surface (skin group) (Figure 2A).
Catheters were inserted inside the rectum, bladder and vagina through natural openings (cavities group). Figure $2 B$ schematically shows the positions of the dosimeters in the cadaver. The bladder was filled with $300 \mathrm{~mL}$ of physiological serum with a urinary catheter.

\section{Radiotherapy procedure}

The dosimetric kV-CT [General Electric ${ }^{\mathrm{TM}}$, Boston (MA), USA] of the pelvic region was performed in a supine position according to the department protocol. The HU scale ranges from $-31,743$ to 31,743 . Axial images were acquired every 

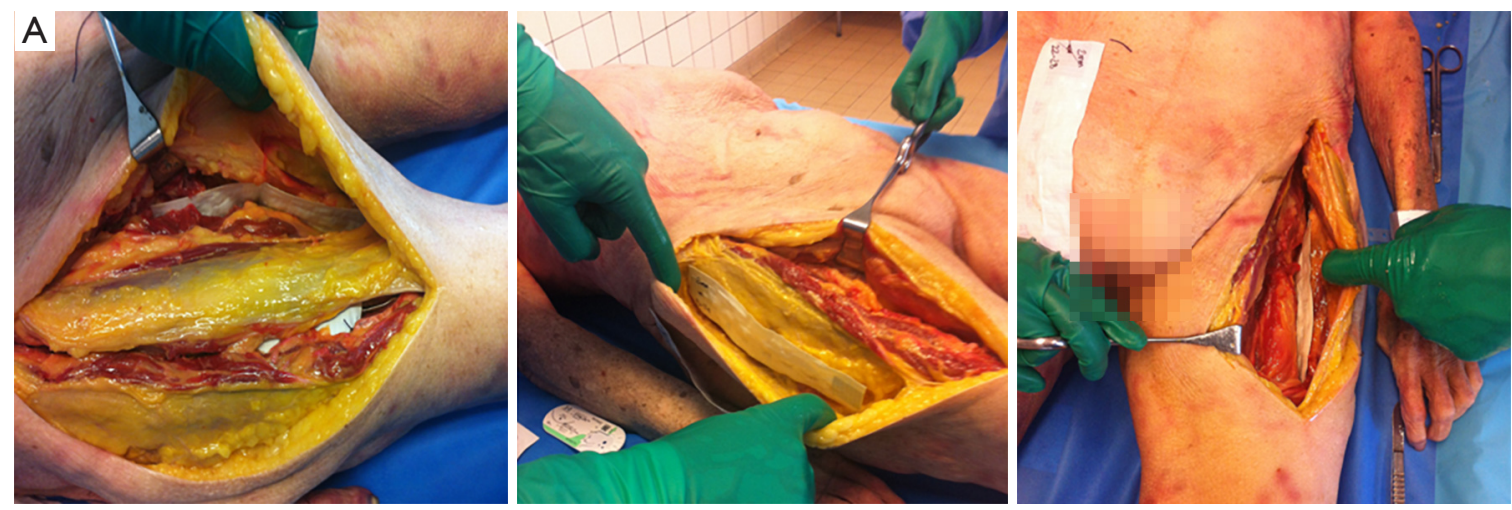

B

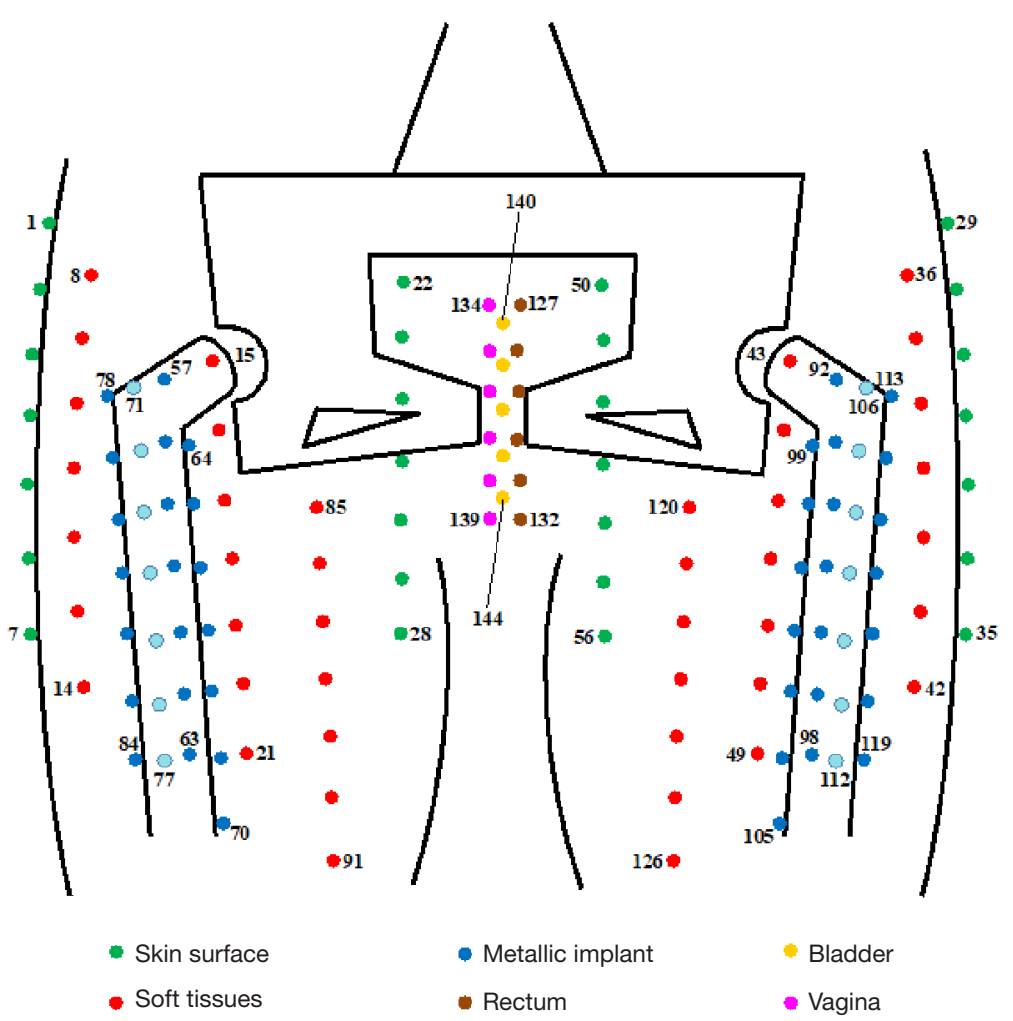

Figure 2 Experimental set up of the alanine dosimeters. (A) Surgical placement of the dosimeters inside the cadaver; (B) scheme of the location of the alanine dosimeters in the cadaver (pelvic region).

\section{$2.5 \mathrm{~mm}$ from L3 to halfway down the femurs.}

The target volumes, OARs, artifacts and dosimeters were delineated on an axial slice of the planning $\mathrm{kV}$-CT with the contouring software FocalSim ${ }^{\mathrm{TM}}$ (Elekta $^{\circledR}$, Stockholm, Sweden). The target volume was defined as a preoperative endometrial cancer. The clinical target volume (CTV) included the external and internal iliac node areas, presacral area, uterus, cervix, left and right parameters and the upper third of the vagina. The planning target volume (PTV) included the CTV with a margin of $5 \mathrm{~mm}$ and corrected at $2 \mathrm{~cm}$ in anteroposterior direction of the upper two-thirds of the uterus. The two hip prostheses, dosimeters, lead markers and artifacts were delineated (Figure $3 A, B, C$ ).

The treatment plan was calculated for a total dose of $50 \mathrm{~Gy}$ in five fractions with the TomoTherapy planning station $^{\mathrm{TM}}$ software (Accuray Inc. ${ }^{\circledR}$, Sunnyvale, CA, USA) using a convolution superposition algorithm. The conversion curve of this TPS was voluntarily limited to 

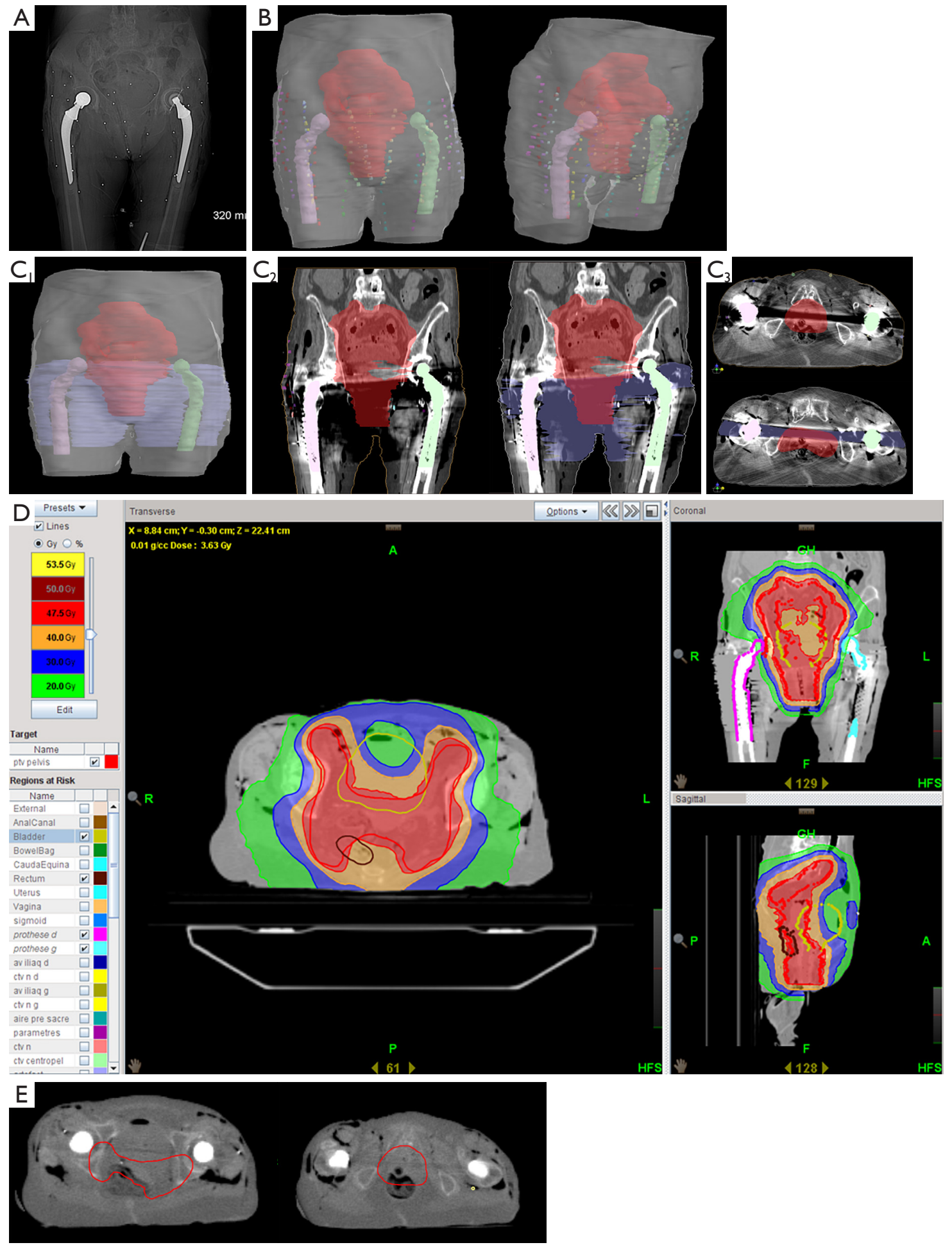

Figure 3 Radiotherapy planning treatment steps. (A) $\mathrm{kV}-\mathrm{CT}$ of the cadaver scout view: hip prostheses and grains of lead on the tabs or catheter ends are hyperdense; (B) delineation of the PTV (red), hip prostheses (pink and green) and each alanine dosimeter (points) on the 3D kV-CT reconstruction; (C) delineation of the artifacts (purple), PTV (red), and prostheses (pink and green): (C1) 3D kV-CT

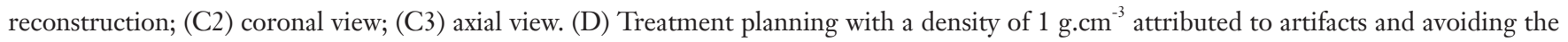
prostheses; (E) MV-CT axial view with the PTV in red. 
Table 1 Differences between the calculated doses and measured doses with and without temperature correction

\begin{tabular}{|c|c|c|c|c|}
\hline Dosimeters groups & $\begin{array}{c}\text { Calculated doses - unadjusted } \\
\text { measured doses (Gy) }\end{array}$ & $P$ value & $\begin{array}{l}\text { Calculated doses - temperature } \\
\text { adjusted measured doses (Gy) }\end{array}$ & $P$ value \\
\hline Global & $0.80(\mathrm{SD} \pm 0.76)$ & 0.0049 & $0.77(\mathrm{SD} \pm 0.74)$ & $<0.001$ \\
\hline Bones group & $0.28(S D \pm 0.35)$ & $<0.001$ & $0.27(\mathrm{SD} \pm 0.35)$ & $<0.001$ \\
\hline Soft tissues group & $0.73(\mathrm{SD} \pm 0.58)$ & $<0.001$ & $0.71(\mathrm{SD} \pm 0.56)$ & $<0.001$ \\
\hline
\end{tabular}

1,994 HU (2.71 ${\mathrm{g} . \mathrm{cm}^{-3}}^{-3}$ to limit the risk of overdose due to repositioning uncertainty. The irradiation beam design avoided the hip prostheses, and a density of $1 \mathrm{~g} \cdot \mathrm{cm}^{-3}$ was attributed to the artifacts previously delineated. Figure $3 D$ represents the dose distribution assessed from the planning treatment. The dose constraint policy of the department was respected. A minimum of $98 \%$ of the PTV received $\geq 95 \%$ of the prescription dose, and hot spots received a maximum of $102.7 \%$ of the prescribed dose.

The irradiation was performed with TomoTherapy HiArt $^{\circledR}$ (Accuray Inc. ${ }^{\circledR}$, Sunnyvale, CA, USA) at a dose of 10 Gy in a single fraction with $6 \mathrm{MV} \mathrm{X}$-ray beams and a dose rate of $8.6 \mathrm{~Gy} / \mathrm{min}$. A MV-CT was performed to check and correct cadaver repositioning and was matched with $\mathrm{kV}-\mathrm{CT}$ images before the treatment (Figure 3E). MV-CT was not used to calculate any dose distributions. The location of the tabs and catheters on the MV-CT was delineated, and their position on the MV-CT was compared to their location on the dosimetric $\mathrm{kV}$-CT to measure their possible motion during the cadaver set-up. Hence, adaptive dosimetry was performed to compare the mean calculated dose of each tab or catheter from the $\mathrm{kV}-\mathrm{CT}$ in one instance and from the MV-CT in another instance.

During irradiation, the cadaver temperature was monitored using a thermometer (Dostmann Eletronic ${ }^{\mathrm{TM}}$ ) and remained at $16.1^{\circ} \mathrm{C}$.

The dosimeter tabs and catheters were collected surgically. Three dosimeters out of a total of 143 positioned dosimeters were altered during the study and were no longer usable for the analysis.

\section{Statistics}

Quantitative variables were expressed as the mean and standard deviation (SD). A paired-sample Student's $t$-test was performed when the distribution of the differences was Gaussian (assessed by Shapiro-Wilk test), or a paired
Wilcoxon's rank test was conducted in the opposite case.

\section{Results}

\section{Doses on the $k V-C T$ and the $M V-C T$}

No statistical differences were found between the calculated mean doses in the $\mathrm{kV}-\mathrm{CT}$ and the adaptative dosimetry in the MV-CT in the bones group ( $\mathrm{P}=0.10)$, soft tissues group $(\mathrm{P}=0.25)$, skin group $(\mathrm{P}=0.25)$ and cavities group $(\mathrm{P}=0.37)$. The mean dose variation of all dosimeters doses was $1.2 \%$ (Table S1).

\section{Calculated and measured doses}

The global analysis of all dosimeters showed a significant difference between TPS-calculated doses and alaninedosimeter-measured doses $(\mathrm{P}<0.001)$ (Figure S1). Table 1 shows the difference between the calculated and measured doses with and without the temperature corrective coefficient. The mean measured corrected doses were $21.9 \%, 17.0 \%$, $21.2 \%, 33.0 \%$ and $19.0 \%$ lower than the mean calculated doses corresponding to an absolute difference of $0.77,0.27$, $0.71,1.15$ and 1.88 Gy for the global group, bone group, soft tissues group, skin group and cavities group, respectively.

\section{Discussion}

To the best of our knowledge, no previous study has compared measured doses and planned doses for radiation treatment in the pelvic area of a human cadaver with hip prostheses. Few previous studies on the human body have been performed $(8,9,12,13)$. The scarcity of these experiments can be explained by ethical and moral reluctance. In some series, comparisons between calculated and measured doses show comparable results $(8,9)$. Wagner et al. analyzed an air balloon covered with alanine 
dosimeters placed in the rectum of three patients treated for prostate cancer. In the patient with a hip prosthesis, the TPS overestimated the dose for the anterior rectal wall by $11 \%$ and underestimated the dose for the posterior rectal wall by $7 \%$ (12). In a more recent study, Wagner et al. showed a mean absolute deviation of 0.08 Gy for their first group of eight patients but a mean absolute deviation of minus 5.13 Gy for the second group of seven patients comparing alanine-detector-measured doses and TPScalculated doses of the urethra during brachytherapy. The measured dose depended on the correct application of alanine detectors in the urethra (13). In our experiment, the TPS seemed to overestimate the delivered dose by an average of $15.8 \%$ and ranged from $4.0 \%$ to $19.4 \%$, which corresponds to an absolute dose of 0.4 to $1.94 \mathrm{~Gy}$, according to the position of the dosimeters in the rectum.

The results of this current study showed a significant discrepancy between TPS-calculated doses and alanine dosimeter measured doses. Indeed, the measured doses ranged from $17.0 \%$ to $33.0 \%$ lower than the calculated doses, although the planning dosimetry avoided the prostheses, and a density of 1 g.cm-3 was attributed to artifacts. Using water tanks or phantoms miming hip MIs, some authors showed a dose increase on the first millimeters of the tissue-implant interface from $15 \%$ to $25 \%(1,14-16)$ and a dose attenuation along and in the shadow of the MI from $10 \%$ to $64 \%(15,17-21)$. The discrepancies between values depend on the components and internal structure of the implant (21), the electron density of material, the $Z$ number $(1,22)$, the dimensions of the implant (19) and ballistic features of the radiation beam (23). Near the prostheses, the current results are different since the calculated doses were lower than the measured doses. Although the components and other characteristics of the prostheses were unknown, some reasons could explain these differences: (I) the radiation beam did not cross the prostheses, and their components had not been sufficiently activated to produce radiations; (II) the path traced by this potential-induced radiation might be too short to be measured by the dosimeters placed $5 \mathrm{~mm}$ away from the prostheses and (III) with the conversion curve of the TPS voluntary limited to 1,994 HU, the measured doses were probably lower than calculated doses. However, dosimeter dose measurements close to the bones group showed the smallest difference with the calculated doses, which could suggest an increase of dose in the area in contact with the prostheses, counteracting the observed discrepancies between the calculated and measured doses.

The use of a frozen human cadaver seemed to be more appropriate than a formaldehyde preserved one. Indeed, formaldehyde could have had an impact on the human tissues physical properties such as attenuation coefficient. Since the aim of this study was to reproduce clinical irradiation conditions, a frozen cadaver appeared to be the best experimental subject.

To improve accuracy in the calculations, TPS should integrate the electron density of the different MI components, their size and form and whether the MI is crossed by the beam (23). However, the real composition of the prosthesis remains questionable since the literature description is not always complete and because of trade secrets (1). Patients with MIs could have an information card with the implant characteristics that could be inputted into the TPS to enhance the calculations (5). Nevertheless, today, TPS used in clinical practice do not always integrate $\mathrm{HU}$ corrections as accurately as required since they are not calibrated for such high densities. Moreover, precise prosthesis densities are not used in current practice because in the case of patient repositioning errors, a risk of overdose could be observed beyond the prosthesis. The specifications of the beams, such as their type and their energy, impact the dose distribution in the presence of metallic materials $(1,19,22,24-26)$, and the larger the number of beams is, the lower the discrepancies between the calculated and measured doses (16).

Alanine dosimeters were used to measure the received dose on different parts of the cadaver. The alanine dosimeter results could be criticized because of the potential lack of precision of this dose measurement technique, especially at low doses. To avoid this limitation, the alanine dosimeters were pre-irradiated with $2 \mathrm{~Gy}$, and the cadaver dose was $10 \mathrm{~Gy}$ in one fraction. A large pre-irradiation dose could possibly improve the detection sensitivity of the dosimeters, as recently shown by Geso et al., who used $30 \mathrm{~Gy}$ (27).

As temperature influences in the dose measurements (12), we corrected the measured dose by a corrective factor. However, we had no information about repeated variations of temperature on the free radical stability in alanine dosimeters.

Spectrometer calibration is another issue $(23,24)$. However, to keep the results reliable, the spectrometers were calibrated and checked before the experiment, and measurements were controlled in two spectrometers. The alanine dosimeters had the same mass, came from the same 
batch and were measured twice. The reproducibility of the dosimeter locations between the dosimetric $\mathrm{kV}-\mathrm{CT}$ and the irradiation with the MV-CT was checked. Indeed, cadaver positioning cannot explain the discrepancies in doses between the TPS calculations and alanine dosimeter measurements (data not shown).

To verify the discrepancies between the calculated and measured doses due to dosimeter delineation, we collected the calculated doses in a sphere with two-millimeter margins around each initial dosimeter contour. The mean calculated dose after adding $2 \mathrm{~mm}$ margins was significantly different compared to the mean initial calculated dose $(\mathrm{P}=0.005)$, but the relative difference was very low [0.002 Gy $(0 ; 0.124)]$ compared to the differences in dose between calculated and measured doses. The dosimeters' delineation could not explain the differences observed between the calculated and measured doses.

Other types of dosimeters could be used instead of alanine dosimeters like TLD (thermoluminescent dosimeters). Despite a lower dose accuracy with alanine dosimeters compared with TLD [5\% versus $2 \%$ respectively (28)], alanine dosimeters remained less expansive, more available, more applicable, more convenient to use with a readout simplicity and had a good linearity with integrated dose.

Some authors showed that the dose measurement in alanine dosimeters has an uncertainty of approximately $5 \%$ $(11,23,29,30)$. Ciesielski et al. evaluated the in vivo alanine dosimeter accuracy through comparing measured and planned doses. The dose uncertainty ranged from $6.6 \%$ at 0.5 Gy to $3.2 \%$ at 2 Gy (24). Helt-Hansen et al. evaluated the dose accuracy of alanine dosimeters and found that the dose accuracy was $>2 \%$ for doses below 4 Gy, less than $2 \%$ between 4 and 10 Gy and $<1 \%$ for doses above 10 Gy (31). In our experiments, the spectrometer found an uncertainty of $2 \%$ for alanine dosimeters receiving more than $6 \mathrm{~Gy}$ and $3.6 \%$ for dosimeters receiving approximately $1 \mathrm{~Gy}$.

\section{Conclusions}

This study showed that the presence of high $\mathrm{Z}$ materials led to delivering doses potentially $17.0 \%$ to $33.0 \%$ below the expected TPS-calculated doses. The TPS seemed to overestimate the calculated dose. The alanine dosimeter protocol requires precision. Knowledge about the precision of the TPS algorithm and MI characteristics is needed to decrease the uncertainties in dose calculations. Other studies on the effects on MIs in radiotherapy are required to improve the irradiation treatment accuracy.

\section{Acknowledgments}

We acknowledge the Morphology and Normal Anatomy Institute of the University.

Funding: None.

\section{Footnote}

Conflicts of Interest: All authors have completed the ICMJE uniform disclosure form (available at http://dx.doi. org/10.21037/cco.2020.01.09). The authors have no conflicts of interest to declare.

Ethical Statement: The authors are accountable for all aspects of the work in ensuring that questions related to the accuracy or integrity of any part of the work are appropriately investigated and resolved. This study received institutional ethics board approval from the research committee of the comprehensive cancer center.

Open Access Statement: This is an Open Access article distributed in accordance with the Creative Commons Attribution-NonCommercial-NoDerivs 4.0 International License (CC BY-NC-ND 4.0), which permits the noncommercial replication and distribution of the article with the strict proviso that no changes or edits are made and the original work is properly cited (including links to both the formal publication through the relevant DOI and the license). See: https://creativecommons.org/licenses/by-nc-nd/4.0/.

\section{References}

1. Reft C, Alecu R, Das IJ, et al. Dosimetric considerations for patients with HIP prostheses undergoing pelvic irradiation. Report of the AAPM Radiation Therapy Committee Task Group 63. Med Phys 2003;30:1162-82.

2. Gschwind R, Buffard E, Masset H, et al. Dosimetric influence of hip prosthesis during radiotherapeutic treatment. Cancer Radiother 2008;12:102-9.

3. Son SH, Kang YN, Ryu MR. The effect of metallic implants on radiation therapy in spinal tumor patients with metallic spinal implants. Med Dosim 2012;37:98-107.

4. Chapman D, Smith S, Barnett R, et al. Optimization of tomotherapy treatment planning for patients with bilateral hip prostheses. Radiat Oncol 2014;9:43.

5. Le Fèvre C, Buffard E, Antoni D, et al. [Consequences of prosthesis on quality of the radiation therapy]. Cancer Radiother 2016;20 Suppl:S259-263. 
6. Carolan M, Dao P, Fox C, et al. Effect of hip prostheses on radiotherapy dose. Australas Radiol 2000;44:290-5.

7. Ng WL, Brunt J, Temple S, et al. Volumetric modulated arc therapy in prostate cancer patients with metallic hip prostheses in a UK centre. Rep Pract Oncol Radiother 2015;20:273-7.

8. Grams MP, Fong de Los Santos LE, Antolak JA, et al. Cadaveric verification of the Eclipse AAA algorithm for spine SBRT treatments with titanium hardware. Pract Radiat Oncol 2016;6:131-41.

9. de Mello-Filho FV, Auader M, Cano E, et al. Effect of mandibular titanium reconstructive plates on radiation dose. Am J Otolaryngol 2003;24:231-5.

10. Anton $M$. Development of a secondary standard for the absorbed dose to water based on the alanine EPR dosimetry system. Appl Radiat Isot 2005;62:779-95.

11. Anton M. Uncertainties in alanine/ESR dosimetry at the Physikalisch-Technische Bundesanstalt. Phys Med Biol 2006;51:5419-40.

12. Wagner D, Anton M, Vorwerk H, et al. In vivo alanine/ electron spin resonance (ESR) dosimetry in radiotherapy of prostate cancer: a feasibility study. Radiother Oncol 2008;88:140-7.

13. Wagner D, Hermann M, Hille A. In vivo dosimetry with alanine/electron spin resonance dosimetry to evaluate the urethra dose during high-dose-rate brachytherapy. Brachytherapy 2017;16:815-21.

14. Buffard E, Gschwind R, Makovicka L, et al. Study of the impact of artificial articulations on the dose distribution under medical irradiation. Nucl Instrum Methods Phys Res 2005;229:78-84.

15. Erlanson M, Franzén L, Henriksson R, et al. Planning of radiotherapy for patients with hip prosthesis. Int J Radiat Oncol Biol Phys 1991;20:1093-8.

16. Ding GX, Yu CW. A study on beams passing through hip prosthesis for pelvic radiation treatment. Int J Radiat Oncol Biol Phys 2001;51:1167-75.

17. Schild SE, Robinow JS, Casale HE, et al. Radiotherapy treatment planning for prostate cancer in patients with prosthetic hips. Med Dosim 1992;17:83-6.

18. Alecu R, Alecu M, Loomis T, et al. Traditional and MLC based dose compensator design for patients with hip prostheses undergoing pelvic radiation therapy. Med Dosim 1999;24:33-7.

19. Eng TY. Dose attenuation through a titanium alloy hip prosthesis. Med Dosim 2000;25:7-8.

20. Spadea MF, Verburg JM, Baroni G, et al. The impact of low- $Z$ and high- $Z$ metal implants in IMRT: a Monte Carlo study of dose inaccuracies in commercial dose algorithms.
Med Phys 2014;41:011702.

21. Hazuka MB, Stroud DN, Adams J, et al. Prostatic thermoluminescent dosimeter analysis in a patient treated with 18 MV X rays through a prosthetic hip. Int J Radiat Oncol Biol Phys 1993;25:339-43.

22. von Voigts-Rhetz P, Anton M, Vorwerk H, et al. Perturbation correction for alanine dosimeters in different phantom materials in high-energy photon beams. Phys Med Biol 2016;61:N70-9.

23. Alves GG, Kinoshita A, Oliveira HF de, et al. Accuracy of dose planning for prostate radiotherapy in the presence of metallic implants evaluated by electron spin resonance dosimetry. Braz J Med Biol Res 2015;48:644-9.

24. Ciesielski B, Schultka K, Kobierska A, et al. In vivo alanine/ EPR dosimetry in daily clinical practice: a feasibility study. Int J Radiat Oncol Biol Phys 2003;56:899-905.

25. Biggs PJ, Russell MD. Effect of a femoral head prosthesis on megavoltage beam radiotherapy. Int J Radiat Oncol Biol Phys 1988;14:581-6.

26. Bergstrand ES, Shortt KR, Ross CK, et al. An investigation of the photon energy dependence of the EPR alanine dosimetry system. Phys Med Biol 2003;48:1753-71.

27. Geso M, Ackerly T, Lim SA, et al. Application of the "Spiking" method to the measurement of low dose radiation $(\leq 1 \mathrm{~Gy})$ using alanine dosimeters. Appl Radiat Isot 2018;133:111-6.

28. Ismail A. Etude et développement d'un système de dosimétrie in vivo implantable basé sur la radioluminescence du nitrure du gallium GaN. Physique. Université Joseph-Fourier - Grenoble I, 2009 tel-00452623.

29. Castro F, Ponte F, Pereira L. Development of physical and numerical techniques of Alanine/EPR dosimetry in radiotherapy. Radiat Prot Dosimetry 2006;122:509-12.

30. Wagner D, Anton M, Vorwerk H. Dose uncertainty in radiotherapy of patients with head and neck cancer measured by in vivo ESR/alanine dosimetry using a mouthpiece. Phys Med Biol 2011;56:1373-83.

31. Helt-Hansen J, Rosendal F, Kofoed IM, et al. Medical reference dosimetry using EPR measurements of alanine: development of an improved method for clinical dose levels. Acta Oncol 2009;48:216-22.

Cite this article as: Le Fèvre C, Brinkert D, Menoux I, Kuntz F, Antoni D, El Bitar Z, Thiery A, Fabbro R, Noël G. Effects of a metallic implant on radiotherapy planning treatmentexperience on a human cadaver. Chin Clin Oncol 2020;9(2):14. doi: $10.21037 /$ cco.2020.01.09 
Table S1 kV-CT and MV-CT mean calculated doses for each dosimeter tab and catheter

\begin{tabular}{|c|c|c|c|}
\hline Alanine dosimeters tabs and catheters number & Location of alanine dosimeters & kV-CT mean dose (Gy) & MV-CT mean dose (Gy) \\
\hline $1-7$ & Skin surface & 1.60 & 1.51 \\
\hline $8-14$ & Soft tissues & 1.63 & 1.63 \\
\hline $15-21$ & Soft tissues & 2.98 & 2.97 \\
\hline $22-28$ & Skin surface & 5.43 & 5.36 \\
\hline 29-35 & Skin surface & 0.95 & 0.96 \\
\hline $36-42$ & Soft tissues & 1.55 & 1.53 \\
\hline $43-49$ & Soft tissues & 4.10 & 4.10 \\
\hline $50-56$ & Skin surface & 5.60 & 5.47 \\
\hline $57-63$ & Metallic implant/bones & 2.15 & 2.04 \\
\hline $64-70$ & Metallic implant/bones & 0.90 & 0.90 \\
\hline $71-77$ & Metallic implant/bones & 3.97 & 3.97 \\
\hline $78-84$ & Metallic implant/bones & 0.69 & 0.66 \\
\hline $85-91$ & Soft tissues & 5.05 & 5.05 \\
\hline $92-98$ & Metallic implant/bones & 1.87 & 1.86 \\
\hline 99-105 & Metallic implant/bones & 0.64 & 0.64 \\
\hline $106-112$ & Metallic implant/bones & 2.23 & 2.22 \\
\hline $113-119$ & Metallic implant/bones & 0.54 & 0.54 \\
\hline $120-126$ & Soft tissues & 4.54 & 4.53 \\
\hline $127-133$ & Rectum & 9.89 & 9.89 \\
\hline $134-139$ & Vagina & 9.95 & 9.89 \\
\hline $140-144$ & Bladder & 10.04 & 9.87 \\
\hline
\end{tabular}

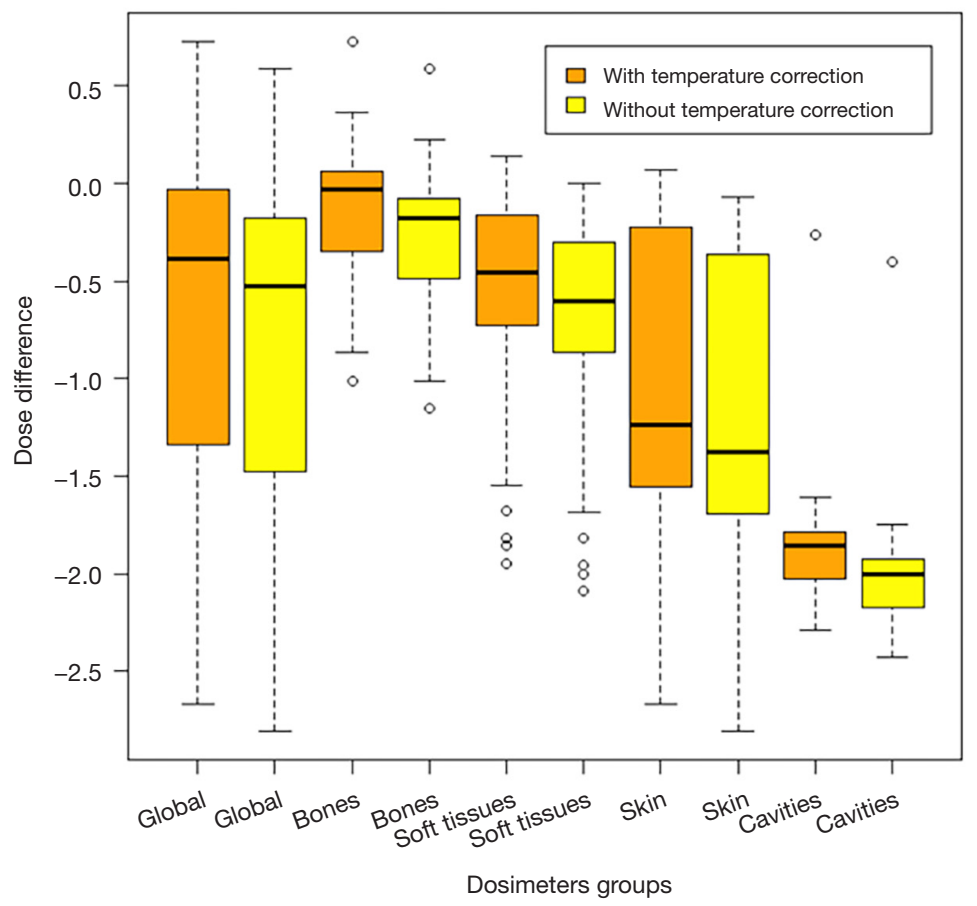

Figure S1 Box-plot representing the difference between the measured dose and calculated dose for each dosimeter group with and without temperature corrective factors. 